\title{
The EANS specialist of neurosurgery diploma
}

\author{
Torstein R. Meling ${ }^{1}$
}

Received: 27 December 2019 / Accepted: 7 January 2020 / Published online: 16 January 2020

(C) Springer-Verlag GmbH Austria, part of Springer Nature 2020

\section{Background}

The EANS training courses are renowned for their excellence since their inception in the 1970s and remain the core business of the EANS [1-3]. It has been a great honor to serve as Training Committee (TC) chairman 2017-2019 and a great privilege to work with Europe's most eminent teachers. Together, we have inspired and taught a generation of very competent trainees that will become leaders of European neurosurgery in the future. We continued to structure, develop, and improve the training courses. The teaching was modernized, and we recently introduced endovascular simulators and demonstrations of endoscopic surgery. Due to an increasing demand, we now have three annual training courses and a record number of graduates each year [3].

However, basic training not only consists of a theoretical training but also a development of practical skills. During my tenure in the EANS TC from 2009 to 2019, it became apparent that there was an unmet need for basic training of neurosurgical skills [4]. We started the EANS Hands-On Course in Lyon back in 2012, and it has been an extraordinary success. Regretfully, a recent article in Acta Neurochirurgica demonstrated a strong decline in surgical cases performed by European neurosurgical trainees during residency, especially for trainees graduating after introduction of the European work-time directive (WTD 2003/88/EC) [5].

\section{Status quo}

There is a need for improved training of practical skills, and the EANS is ideally suited to provide such training, building

This article is part of the Topical Collection on Neurosurgery Training

Torstein R. Meling

torsteinrmeling@gmail.com

1 Hôpitaux Universitaires de Genève, Rue Gabrielle Perret-Gentil 4, 1211 Geneva, Switzerland on our vast experience from the past 15 hands-on courses in Lyon. Over the years, we realized that there was a great need for structured, faculty-intense practical teaching, and secondly, that for some participants, the Lyon course was too advanced, whereas for others, it could have been more in-depth. Therefore, the TC created structured curricula for the practical training and we established four new courses in cranial and spinal surgery for residents in their 1-3rd year (step I), as well as for 4-6th year (step II). The inaugural step I courses in cranial and spinal surgery were held in Brno in 2017 and in Geneva in 2019, respectively. The inaugural step II courses in cranial and spinal surgery were held in Geneva and Lyon, respectively, in 2019.

The EANS Step I and II Hands-On Courses complement the theoretical training of the EANS Training Courses (Fig. 1) and are designed to provide valuable experience for residents at all training levels. The EANS Step I and II Hands-On Courses seek to improve the basic neurosurgical skills training due to a coherent and structured curriculum. The step I courses concentrate on basic surgical anatomy, and the procedures we expect the residents should master after their 3rd year of training. The step II courses repeat the anatomy, but go more indepth, and teaches much more advanced procedures. It summarizes what we expect residents to master after their 6 th year of training. Each course generally has 20 participants and up to 10 faculty members, making them very faculty-intense. Furthermore, the up-front teaching is minimized, allowing for maximum time in the lab. Finally, as with the EANS Training Courses, the EANS Hands-On Courses emphasize social interaction and networking events.

\section{EANS diploma of neurosurgery}

The EANS Hands-On Courses will improve the basic training of neurosurgical skills due to a coherent and structured curriculum. A structured training program and the quality teaching by our renowned EANS faculty will increase the interest in our courses and might even lead to a demand for more step I 
Fig. 1 EANS portfolio for basic courses
Vascular

Tumour

Spine \& Peripheral nerve

Functional \& trauma

Basic training
Knowledge

\section{Skills}

Cranial Step I

Spinal Step I

Cranial Step II

Spinal Step II

and II courses (analogous to the training courses that went from one to two cycles per year in 2002 and up to three cycles per year in 2017).

A formalized EANS Specialist of Neurosurgery Diploma may be a small, but important step towards a more uniform European training, in line with the objectives of the UEMS and the JRAAC.

We look for benefactor(s) or endowments from neurosurgical colleagues who are willing to sponsor a monetary prize along with the diploma and who will have the privilege of naming the prize (analogous to the Braakman Prize).

This initiative is in line with a strategy to build the EANS bottom-up, by engaging young trainees in EANS activities early during their careers; by offering them valuable training; and, finally, by offering them a formal EANS diploma as a sort of "black belt of neurosurgery" upon completing the eight courses of basic training.

Lastly, this initiative will strengthen our position as the premiere agent of neurosurgical training in Europe.

A formal proposal to establish a special diploma was made to the EANS board during EANS2019 in Dublin. The proposal was adopted and the "EANS Specialist of Neurosurgery Diploma" will be presented during the EANS congresses by the EANS president to those who have participated in all four theoretical courses and all four practical courses.

\section{References}

1. Gjerris F (1995) Future EANS training programs and certification. Childs Nerv Syst 11(1):55-56

2. Guidetti B (1987) European courses of neurosurgery and other training and postgraduate education activities of the European Association of Neurosurgical Societies. Acta Neurochir 86(3-4): $115-128$

3. Lo WB (2017) Course review: European Association of Neurosurgical Societies (EANS) training course. Acta Neurochir 159(5):755-756

4. Stienen MN, Gempt J, Gautschi OP, Demetriades AK, Netuka D, Kuhlen DE et al (2017) Neurosurgical resident training in Germany. J Neurol Surg A Cent Eur Neurosurg 78(4):337-343

5. Stienen MN, Bartek J Jr, Czabanka MA, Freyschlag CF, Kolias A, Krieg SM et al (2019) Neurosurgical procedures performed during residency in Europe-preliminary numbers and time trends. Acta Neurochir 161(5):843-853

Publisher's note Springer Nature remains neutral with regard to jurisdictional claims in published maps and institutional affiliations. 